\title{
ON THE THEORY OF NP-COMPLETE PROBLEMS
}

S.V. Listrovoy,

Doctor of technical sciences,

professor of Ukrainian State Academy of Railway Transport, Kharkov.

\section{ABSTRACT}

om1@yandex.ru

It is shown that the theory of NP -complete problems can't be used for the analysis of optimizing tasks.

\section{Indexing terms/Keywords}

NP-complete problem, polynomial reducibility

\section{Council for Innovative Research}

Peer Review Research Publishing System

Journal: International Journal of Computers \& Technology

Vol 11, No.4

editor@cirworld.com

www.cirworld.com, member.cirworld.com 


\section{INTRODUCTION}

Now seven mathematical problems of the tasks of the millennium included the list one of them are known it is a task about interrelation of classes $P$ and NP. The question of interrelation of classes $P$ and $N P$, is considered now one of the main open questions of modern mathematics and theoretical cybernetics. Founders of this problem are Stephen Arthur Cook the professor of university from Toronto, the winner of an award of Turing and professor Leonid Levin. In Cook's works the concept of of NP-complete problems was entered and is proved that the task "feasibility" known still as a SAT task is a universal $N P$-complete task. Further development of the theory of $N P$-complete tasks was carried out by the professor of the Harward university Richard Meningom Karp. Incentive motive for writing of this short message, were works of the Indian mathematician Vineya Deolalikar and the article "On the Relationship between Classes $P$ and $N P$ " of the Ukrainian professor of Anatoly D. Plotnikov in Journal of Computer Science 8 (7):

1036-1040, 2012. In these works it is proved, the fact of discrepancy of these classes. The purpose of this short message is to show an incorrectness of these attempts of the proof on the basis of results already received by the author in works $[4,5]$ and results received in Lavrov and Zykova's works $[6,7,8]$.

\section{PROBLEM SETTING AND SOLUTION}

The theory of NP-complete problems is constructed for problems of recognition of properties. The problem of recognition $L$ can be considered as consisting of two sets: $D_{i}$ and $Y_{\partial}$, where $D_{i}-$ set of all single problems, and $Y_{\partial}-$ set of problems with the answer "yes", thus $Y_{\partial \subset} \subset D_{i}$. The form of these problems consists of two parts. In the first part the exposition of conditions of the problem in terms of various components is given: sets, networks, numbers etc. In the second part the question assuming one of two answers "yes" or "no" is formulated. Informally, class of NP-complete problems is defined by means of concept of nondeterministic algorithm. Such algorithm consists of two stages: guessing and check. At first, under the set single problem I a guessing of structure $S$ takes place, and further, taking into account statements of problem I, check by the determined algorithm which is ended either by the answer "yes" or "no" is carried out. As it is shown in [1], nondeterministic algorithm solves the problem of recognition $L$, if for any single problem $I \in D_{i}$ two following conditions are met:

1. If $I \in Y_{\partial}$ there is such structure $S$ which guessing leads to that the check stage will be completed by the answer "yes".

2. If $I_{\notin} Y_{\partial}$ there is no such structure $S$ which guessing for I will lead to that the check stage will be completed by the answer «no».

The concept of polynomial "checkability" [1] allows actual selection of a class of NP-complete problems, and in addition, "checkability" for polynomial time does not attract decidability of the problem of recognition for polynomial time.

The problem of recognition $L$ is called NP - complete if $L \in N P$ and any other problem $L$ 'from this class is reduced to $L$ polynomially.

Let's assume that the polynomial algorithm for solution of some NP-complete class problem I is obtained, then according to Cook theorem $[1,2,3]$ it follows polynomial resolvability of all NP-complete class problems as it appears from Cook theorem they are polynomially reduced to each other. However in the study [4] it is shown that Cook theorem is incorrect also a class of NP-complete problems is introduced incorrectly. Therefore, polynomial resolvability of problems called in studies of Cook and Karp $[1,2,3]$ as NP-complete does not follow from polynomial resolvability of the above mentioned problem as far as in the studies [4,5] it is shown that all problems which related to NP-complete class can be divided into subsets of problems within which polynomial reducibility is possible and the hypothesis is stated that polynomial reductions between subsets of separate individual problems only are most likely possible. Therefore it is possible to speak here only about polynomial resolvability of individual problems which can be reduced to the problem I. As it follows from $[1,6,7,8]$ the fact supports the given hypothesis that now the list of NP-complete problems includes more than three thousand problems, and practically all main problems of graph theory inclusively. Then, proceeding from the polynomial reducibility of problems within this class declared by Cook, for solution of all problems of graph theory listed there should be one algorithm for their deciding with some arbitrarily high complexity that is stipulated by their polynomial reducibility to each other, but this contradicts the outcomes obtained in studies of I. A. Lavrov (1963) [8] and A.A.Zykov (1969) [6] where impossibility of construction of such algorithm is shown.

As appears from [4,5] set of objects which are described by impracticable Boolean functions in exponential number of times surpass number of objects which are described by feasible Boolean functions, and properties of polynomial reducibility are by default transferred and to the objects described by impracticable Boolean functions. Thus, Cook's theorem is fair only for objects described by feasible Boolean functions. Thus it must be kept in mind that the number of such objects isn't enough. Now some words about the proof of NP completeness of any task. As shown in studies [1,2,3], polynomial reducibility of the problem of recognition $l_{1}$ to the problem of recognition $l_{2}$ means availability of function $f$ which transforms a subset of problems $D_{i 1}$ into a subset of problems $D_{R 2}\left(D_{i 1} \rightarrow D_{R 2}\right)$, on the basis of some rule $\Pi_{i}$ and, thus, satisfies to two conditions:

1. $f-$ is calculated by a polynomial algorithm;

2. For all $I \in D_{i} l \in Y_{\partial 1}$, when and only when $f(I) Y \in \partial 2$. 
Let's consider three subsets of problems $\left\{I_{i}\right\} ;\left\{Z_{i}\right\} ;\left\{C_{i}\right\}$. Let the problem $I-N P$-is complete and represents the universal problem, and problems $Z$ and $C$ are also NP-complete then, according to that as the class of NP-complete problems is introduced, they should be reduced polynomially one to another and, thus, if the polynomial algorithm for one of them is discovered, there should be polynomial algorithms for all single problems $\left\{I_{i}\right\} ;\left\{Z_{i}\right\} ;\left\{C_{i}\right\}$. As the universal problem can appear any of NP-complete problems, all following reductions should be true

$$
\begin{aligned}
& \left\{I_{i}\right\} \rightarrow\left\{Z_{i}\right\} \rightarrow\left\{C_{i}\right\} ;(1)\left\{I_{i}\right\} \rightarrow\left\{C_{i}\right\} \rightarrow\left\{Z _ { i } ; \text { ; (2) } \{ C _ { i } \} \rightarrow \{ I _ { i } \} \rightarrow \left\{Z_{i} ;\right.\right. \\
& \left\{C_{i}\right\} \rightarrow\left\{I_{i}\right\} \rightarrow\left\{Z _ { i } ; \text { ; (4) } \{ Z _ { i } \} \rightarrow \{ C _ { i } \} \rightarrow \left\{I_{i} ; \text {; (5) }\left\{Z_{i}\right\} \rightarrow\left\{I_{i}\right\} \rightarrow\left\{C_{i}\right\} .\right.\right.
\end{aligned}
$$

In addition, there are rules $\Pi_{i z}$ and $\Pi_{z c}$ which permit to reduce problems $I_{p} \rightarrow Z_{p}$ and, thus, $\left\{I_{p}\right\} \in Y_{\partial i}$ and problems $Z_{p} \rightarrow C_{p}$ and, in this respect, $\left\{Z_{p}\right\} \in_{\partial z}$, i.e. transformation rules $\Pi_{i z}$ and $\Pi_{z c}$ satisfy to conditions of polynomial reducibility 1 and 2. Let's consider when structures $S$ are such that they generate set of single problems $\{Z\}$ which by its potency exceeds set of single problems \{\} . If the subset \{\} contains $n$ single problems, and sets $\{Z\}$ and $\{C\}$ contain $n+k$ single problems each, then for some subset of problems $\left\{Z_{n+1}, Z_{n+2}, \ldots, Z_{k}\right\}$ we cannot put in correspondence any problem from $\left\{I_{i}\right.$. Therefore, the reductions (1) and (2) are possible for all problems, and reductions (3), (4), (5) and (6) are possible not for all problems, they are not possible for problems $\left\{C_{n+1}, C_{n+2}, \ldots, C_{k}\right\}$ and $\left\{Z_{n+1}, Z_{n+2}, \ldots, Z_{k}\right\}$, and, it means in this case, that the statement about all NP-complete problems are polynomially reduced to each other, is not fulfilled. Thus, the concept of the NP-complete problem requires an improvement. In order that the NP-complete problem was universal and reduced in any directions within a class, it is necessary that there was a one-to-one correspondence between all single problems $\left\{I_{i} ;\left\{Z_{i}\right\} ;\left\{C_{i}\right\}\right.$, i.e. for any pair of single problems there should be the direct and inverse polynomial reduction defined by conditions 1 and 2 .

Thus, if we have subsets of problems $\left\{I_{i} ;\left\{Z_{i}\right\} ;\left\{C_{i}\right\}\right.$, and the potency of set of single problems $\left\{I_{i}\right\}$ differs from a potency of sets of problems $\left\{Z_{i}\right\}$ and $\left\{C_{i}\right\}$, then to prove that some problem $I$ is NP-complete, it is not enough to show that any single problem $\left\{I_{i}\right.$ is polynomially reduced to set of problems $\left\{Z_{i}\right\}$ and $\left\{C_{i}\right\}$, i.e. conditions 1 and 2 are satisfied as it was made in the proof of NP-completeness of the "satisfiability" problem in Cook's and Carp's studies, but thus it is necessary to show, that there are also problems $\left\{I_{n+1}, I_{n+2}, \ldots, I_{k}\right\}$, which are polynomially reduced to problems $\left\{C_{n+1}, C_{n+2}\right.$, $\left.\ldots, C_{k}\right\}$ and $\left\{Z_{n+1}, Z_{n+2}, \ldots, Z_{k}\right\}$, and "checkability" of these recognition problems should remain possible for polynomial time.

\section{CONCLUSIONS}

The problem about correlation of classes $P$ and NP set by Cook and entered into the Millennium problems list is the simply incorrectly set mathematical problem therefore it is no wonder that it was possible to nobody to solve it. Therefore the problem should be eliminated from the Millennium problems list as scientists spend precious time for its solution to this day, studies of Indian mathematician Viney Deolalikar and paper "On the Relationship between $P$ and NP Classes" of Ukrainian professor Anatoly D. Plotnikov in Journal of Computer Science 8 (7: 1036-1040), 2012 testify to it, i.e. presence of the given problem at the Millennium problems list thwarts further progress of mathematics. It is necessary to note also, that all outcomes in the theory of algorithms based on the "total" reducibility in NP-complete class problems declared by Cook should be revised seriously.

\section{REFERENCES}

1. Gary M., Johnson D. Computing machines and hard problems. - M: Mir, 1982. - $336 \mathrm{p}$.

2. Cook S.A. Complexity of procedures of a conclusion of theorems. - Cybernetic collection of a new series, vol.12. Moscow.: Mir, 1975.-P 5 - 15.

3. Karp R.M. Reducibility of combinational problems. - Cybernetic collection of a new series, vol. 12. - Moscow: Mir, 1975.-P16-38.

4. Listrovoy S.V. On Correlation of $P$ and NP Classes//I.J.Modern Education and Computer Science, 2012, 3, 21-27.

5. Listrovoy S.V. "About polynomial reducibility in class NP" Ukrainian Mathematical Congress -2009, Algebra and Number Theory. http://www.imath.kiev.ua/

6. A.A. Zykov, Finite graphs theory//Novosibirsk: Nauka, 1969, p. 542

7. A.A. Zykov. Elements of graph theory//Moscow: Nauka, 1987, p. 381.

8. I.A. Lavrov. Effective inseparability of a set of identically true and a set of finitely refutable formulae of some elementary theories. Algebra and logics (Materials of the workshop of Institute of Computational Mathematics and Mathematical Geophysics of Siberian Branch of the USSR Academy of Sciences) 2 (1963), No.1, 5-18. RzhMath 1964, 1 A112.

Listrovoy Sergey Vladimirovich, doctor of technical sciences, professor of Ukrainian State Academy of

Railway Transport, Kharkov. In 1972 has finished high military command engineering school in Kharkov. The

Area of the scientific studies of the problem to discrete optimization and graph theory and their use to analysis of the computing systems and networks. 